\title{
Free electron laser in the UV
}

\author{
M.E. Couprie \\ CEA DSM SPAM, 91191 Gif-sur-Yvette, France \\ and \\ LURE, Bât. 209D, 91405 Orsay cedex, France
}

Free Electron Lasers (FEL) result from the amplification of the light emitted by an ultrarelativistic electron beam passing through the magnetic field of an undulator, stored in an optical cavity. They constitute coherent tunable sources covering from the far infra-red to the UV, depending on the type of accelerator.The UV range is achieved mainly with storage ring FELs, as on VEPP3, Super-ACO, UVSOR and NIJI-4. The temporal structure is a series of picosecond light pulses, at a high repetition rate, resulting from that of the electron beam. The Super-ACO FEL was first used for a time-resolved fluorescence experiment in biology, demonstrating the high quality and stability of such a source. Pump probe experiments coupling FEL and synchrotron radiation, naturally synchronized, are under way. A first result just has been obtained in semi-conductor physics, where the surface photovoltage effect induced by the Super-ACO FEL has been probed by high resolution photoemission. Recently, the oscillation was also obtained in the UV with a linear accelerator at Los Alamos, gathering several technological challenges. A review of the characteristics of the existing facilities will be given, together with a presentation of the projects, and the prospects for the X-ray domain.

\section{INTRODUCTION}

\subsection{FEL principle}

In a Free Electron Laser, a relativistic electron beam interacts with the sinusoidal permanent magnetic field of an undulator (of spatial period $\lambda_{0}$ and peak magnetic field $\mathrm{B}_{0}$ ) and then emit synchrotron radiation at the wavelength $\lambda$ and its harmonics:

$$
\lambda=\lambda_{0}\left(1+K^{2} / 2\right) / 2 \gamma^{2}
$$

$K$ being the deflection parameter $\left(K=0.94 B_{0}(T) \lambda_{0}(\mathrm{~cm})\right)$ and $\gamma$ the relative energy of the electron beam. This radiation is stored in an optical cavity in order to interact transversally with the electron bunches at each pass in the undulator field, and the optical pulse can be amplified at the cost of the particle kinetic energy, leading to the laser oscillation. Without an optical cavity, an external laser can be amplified according to the same scheme. Coherent harmonics from the FEL or from an external laser can be produced in the amplification medium consisting of the the electron beam in the undulator field.

The FEL can easily be tuned by modifying the magnetic field of the undulator. The laser interaction modifies the energy distribution of the electron beam, leading to the laser saturation (enhancement of energy spread, modification of the resonance condition (1) resulting from an energy change in the beam). The gain of the system increases with longer 
wavelengths, lower beam energy, higher electronic density and longer insertion devices. The laser temporal structure results from that of the electron beam from which it is generated : macropulses constituted of series of micropulses for RF linacs, micropulses for superconducting linacs and storage rings. The polarisation is given by the geometry of the magnetic field of the undulator (linear for a plane undulator, circular for a helicoidal one..). High powers can also be achieved, especially in the infra-red. The drawbacks, as compared to conventional lasers, of such sources are their cost and size.

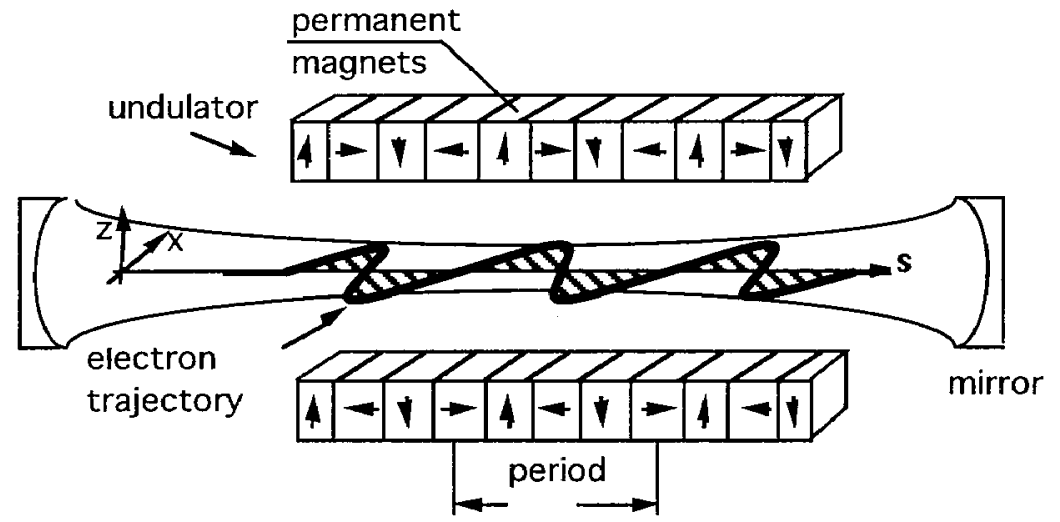

Fig. 1 : Principle of the free electron laser

\subsection{Choice of the type of accelerator}

The FEL spectral range is mainly determined by the energy of the electron beam, and consequently by the accelerator type. For instance, far infra-red corresponds to Van de Graff accelerators, induction linac and microtrons (1-5 MeV), Infra-red to linear accelerator (10-200 MeV) and the UV is well adapted to storage ring $(0.2-1.5 \mathrm{GeV})$, or to linacs with very short period undulators. As the gain becomes smaller with increasing energy, storage rings are generally preferable to linear accelerators, because of their good beam quality.

The first FEL has been operated in the infra-red (IR) on a linear accelerator in Stanford (USA) in 1977 [1] and more than 15 IR facilities are now worldly spread and currently dedicated to users [2]. The first storage ring (SR) FEL, was only achieved in 1983 on ACO at Orsay [3] in the visible and in the UV in 1988 on VEPP3 [4]; the first application experiments, only starting now [5] on the Super-ACO FEL [6], are possible because of its good stability and duradtion $(>10 \mathrm{~h})$.SR FELs represent nevertheless the easiest way for the UV-VUV range, and can be part of to synchrotron radiation centers, thus reducing their cost.

\section{STORAGE RING FEL}

\subsection{Generalities}

\subsubsection{Storage ring specificities}

On a storage ring, the recirculation of the electrons insures a high beam quality and stability, with, especially a small energy spread and a high electronic density. It is then particularly well adapted for short wavelength FELs. A RF cavity compensates the energy loss by turn by synchrotron radiation, and bunches the beam. The use of two RF cavities can allow the shortening of the bunch length, or the stabilization of the beam. The time structure is pulsed at high frequency, resulting from the multipass of the electron bunches. For rather low beam energy, the lifetime is mainly determine by the Touschek effect, getting smaller when the electronic density is increased and the beam energy is lowered.

\subsubsection{General situation}

SR FEL activity started actively in Orsay (France) with the first oscillation in the visible on ACO [3] continued on Super-ACO [6], and in Novosibisk on VEPP 3 [4] with still the shortest wavelength, i.e. $240 \mathrm{~nm}$. On these facilities were studied in details the 
laser charcteristics and behaviour. An increasing work is now been performed in Japan, with the visible FEL at TERAS (Tsukuba) in 1991 [7], at NIJI-4 (Tsukuba) in 1993, the first dedicated storage ring for FEL[8] and at UVSOR (Okazaki) in 1992 [9]. Various projects are under way, as mentionned in table I. The two dedicated FEL facilities (DELTA [10] and Duke University [11]) are close to get the oscillation and should provide a new generation of SRFELs for users. Other projects are under study (see table 1), as for example, the SOLEIL FEL for providing $1 W$ available average power between 350 and $100 \mathrm{~nm}$ in $10 \mathrm{ps}$ pulses, on a synchrotron radiation facility [12].

Table I : List of UV Storage Ring Free Electron laser Facilities ( $P$ total extracted power, I maximum current of lasing, $\tau$ laser duration)

\begin{tabular}{|c|c|c|c|c|c|c|c|c|c|}
\hline FEL & Location & Date & $\begin{array}{l}\text { Energy } \\
(\mathrm{MeV})\end{array}$ & $\begin{array}{l}\text { Spectral } \\
\text { Range } \\
\text { (nm) }\end{array}$ & $\begin{array}{c}\mathrm{P} \\
\mathrm{mW} / \\
10 \mathrm{~mA}\end{array}$ & $\begin{array}{l}\mathrm{I} \\
(\mathrm{mA} / \\
\text { b. })\end{array}$ & $\tau(h)$ & $\begin{array}{l}\text { Gain } \\
(\%)\end{array}$ & REMARKS \\
\hline VEPP3 & $\begin{array}{l}\text { CEI } \\
\text { Novosibisrk }\end{array}$ & $\begin{array}{l}1978-1994 \\
\text { laser } \quad \mathbf{1 9 8 8}\end{array}$ & 350 & $\begin{array}{l}690-580 \\
460-375 \\
270-240\end{array}$ & $\begin{array}{ll}3 \\
1\end{array}$ & $\begin{array}{l}20 \\
20\end{array}$ & 0.5 & 10 & $\begin{array}{l}\text { different optical } \\
\text { klystron, linewidth } \\
\text { narrowing, shortest } \lambda\end{array}$ \\
\hline Super-ACO & $\begin{array}{l}\text { OTsay } \\
\text { FRANCE }\end{array}$ & $\begin{array}{l}1988-? \\
\text { laser } 1989 \\
\text { UV } 1991\end{array}$ & $\begin{array}{l}600- \\
600 \\
700 \\
800\end{array}$ & $\begin{array}{l}690-606 \\
345-355\end{array}$ & $\begin{array}{l}24 \\
10 \\
180 \\
540\end{array}$ & $\begin{array}{l}45 \\
30 \\
22 \\
25\end{array}$ & $\begin{array}{l}2 \\
5.5 \\
12\end{array}$ & 2 & $\begin{array}{l}\text { short micropulse } \\
\text { first user experiments }\end{array}$ \\
\hline UVSOR & $\begin{array}{l}\text { Okazaki } \\
\text { JAPAN }\end{array}$ & $\begin{array}{l}\text { 1988-? } \\
\text { laser } 1992\end{array}$ & 500 & $\begin{array}{l}488 \\
300\end{array}$ & 1 & 10 & 1 & $\begin{array}{l}0.4 \\
0.7\end{array}$ & $\begin{array}{l}\text { gain improvement with } \\
\text { a 2nd RF cavity }\end{array}$ \\
\hline NIJI-4 & $\begin{array}{l}\text { Tsukuba } \\
\text { JAPAN }\end{array}$ & $\begin{array}{l}\text { l990-? } \\
\text { laser } 1992 \\
\text { UV } 1994\end{array}$ & 240 & $\begin{array}{l}595 \\
353\end{array}$ & low & & 1 & $5-10$ & $\begin{array}{l}\text { first dedicated SR FEL } \\
\text { SR built by Kawasaki } \\
\text { H. Ind. }\end{array}$ \\
\hline DELTA & $\begin{array}{l}\text { Dortmund } \\
\text { GERMANY }\end{array}$ & 1989-? & $\begin{array}{l}500- \\
1500\end{array}$ & $\begin{array}{l}\text { Visible } \\
\text { UV }\end{array}$ & & & & $>10$ & $\begin{array}{l}\text { Dedicated facility } \\
\text { 1995: commissioning }\end{array}$ \\
\hline DUKE & $\begin{array}{l}\text { N- Carolina } \\
\text { USA }\end{array}$ & $\begin{array}{l}1989 \text { tranfer } \\
\text { from Stanfort }\end{array}$ & 1000 & $\begin{array}{l}\text { visible- } \\
\text { VUV }\end{array}$ & & & & $>10$ & $\begin{array}{l}1995 \text { : commisioning } \\
\text { dedicated facility }\end{array}$ \\
\hline KEK & Tsukuba JAP. & \begin{tabular}{l|l}
1991 \\
\end{tabular} & 750 & 177 & & & & 10 & 1992: optical klystron \\
\hline$\overline{\text { FELI }}$ & $\begin{array}{l}\text { Hirakata } \\
\text { JAPAN }\end{array}$ & 1992 & 700 & 200 & & & & & $\begin{array}{l}\text { common installation } \\
\text { with IR LINAC FELs }\end{array}$ \\
\hline NIKHEF & Amsterdam NI & 1993 & 900 & VUV & & & & & non dedicated \\
\hline EUTERPE & Eindhoven NL & 1993 & 400 & XUV & & & & & non dedicated facitity \\
\hline SOLEIL & France & 1993 & 1500 & $350-100$ & $\begin{array}{l}\text { few } \\
\text { W }\end{array}$ & $\begin{array}{l}4 \mathrm{x} \\
5 \mathrm{~mA}\end{array}$ & $>5$ & $>10$ & $\begin{array}{l}2.15 \mathrm{GeV} \text { synchrotron } \\
\text { radiation facility }\end{array}$ \\
\hline SINBAD & Daresbury GB & 1993 & 700 & XUV & & & & & not yet funded \\
\hline
\end{tabular}

\subsubsection{The optical klystron}

The available short straight section for insertion devices on usual storage rings led to short interation region, and then a rather low gain. It was then proposed to increase articifially the gain, with a variety of undulator, called optical klystron [13]. It consists of two undulator sections, separated by a large wiggle of magnetic field, created by the socalled dispersive section. In the first undulator occurs the energy modulation of the particules in the bunch, transformed into a density modulation by the dispersive section, leading to a more coherent emission in the second undulator. The radiation results from the interference from the two undulator spectra, with a modulation rate, $f$, equivalent to the optical constrast of the Young slits. $\mathrm{f}$ scales as $\exp \left(-\left(\sigma_{\gamma / \gamma}\right)^{2}\right), \sigma_{\gamma} / \gamma$ being the energy spread. Optical klystrons can be set-up on usual straight sections of the ring, or on dedicated by-passes, as on the VEPP 3 experiment[4] .

\subsubsection{Gain and saturation}

The gain, in the optical klystron case, is given by:

$\mathrm{G}_{\mathrm{OK}}=1.1210^{-13}\left(\mathrm{~N}+\mathrm{N}_{\mathrm{d}}\right) \mathrm{K}^{2} \mathrm{Lund}^{2} \mathrm{JJ}^{2} f \rho_{\mathrm{e}} \mathrm{F}_{\mathrm{f}} \exp \left(-\tau^{2} / 2 \sigma^{2}\right) / \gamma^{3}$ 
with $\rho_{\mathrm{e}}$ the electronic density, $\mathrm{N}$ the number of periods of the undulator and $\mathrm{Nd}$ the interference order due to the dispersive section, JJ a term of Bessel functions (approximately equal to $\left(2 \mathrm{~K}^{2}+2\right) /\left(4+2 \mathrm{~K}^{2}\right)$ ), $\mathrm{F}_{\mathrm{f}}$ a filling factor representing the transverse overlap between the electron and photon beams, $\tau$ is the detuning term between the optcal and electron pulses, and $\sigma$ is the bunch length. The choice of the beam energy is a compromise between the beam lifetime, the laser power (increasing with $\gamma$ ) and the gain and mirror degradation (decreasing with $\gamma$ ).

The laser interaction leads to an enhancement of the energy spread, related to a bunch lengthening, as on VEPP3. This phenomenon is not really observed on the SuperACO FEL, because there is a competition between the bunch heating and the pehnomena responsible for the anomalous bunch lengthening occuring with higher currrents. In that case, the laser (damps the coherent synchrotron oscillations of the bunches, and stabilizes the beam. The saturation occurs both by the energy spread induced by the laser (in the modulation rate term) and by detuning between the electron and optical pulses (exponential term) [14].

\subsubsection{The optical cavity}

The cavity length is a sub multiple of the ring circumference in order to insure the proper synchronization between the bunches stored in the accelerator, and the optical pulses bouncing between the two mirrors. The transverse overlap requirement of both pulses leads to the choice of the radius of curvature of the mirrors.

The choice of the mirrors is very critical, because they are exposed to the whole radiation spectrum of the undulator. In the case of Super-ACO for example at $800 \mathrm{MeV}$, the undulator power hitting the front mirror is of $20 \mathrm{~W}$, whereas only $1 \mathrm{~W}$ of the laser power is absorbed. In addition, for a fundamental wavelength at $350 \mathrm{~nm}$ (see (1)), half the radiation is emitted below $70 \AA$.

On the present SR FEL experiments, the rather small gain of the system $(10 \%$ maximum) requires the use of multidielectric mirrors in order to keep cavity losses smaller than the gain. When the undulator power is already high (Super-ACO case), the layers are deposited on sapphire substrates because of the high thermal conductibility of this material. It then limits the mirror heating due to synchrotron radition, and the associated mechanical distortion, responsible for a lasing on high order transverse modes. In addition, super-polished substrates are required in order to minimize the scatter losses, and especially for shorter wavelengths. This presents an important technological challenge, particularly for sapphire. The VUV and X ray radiation emitted in the undulator may also lead a to rapid mirror degradation, depending on the deposition technique. Ion Beam Sputtering (degradation rate less than 1\%/A.h), developed for gyrolasers optics, gives more resistant mirrors than the ones produced by simple evaporation technique (40\%/A.h) and even Ion Assisted Deposition (10\%/A.h). The losses level can be recovered by baking and oxygene plasma exposing. In addition, the materials become more absorbant for shorter wavelengths.

\subsection{Characteristics}

\subsubsection{Spectral characteristics}

The spectral range of the FEL is determined by the characteristics of the undulator and by the energy of the accelerator. As illustrated in table I, several SRFELs have achieved the oscillation itn the UV: the shortest wavelength still remains on VEPP 3 around $240 \mathrm{~nm}$, with UVSOR at $300 \mathrm{~nm}$ and Super-ACO and NIJI-4 around $350 \mathrm{~nm}$. The tunability (30 nm on VEPP3, $10 \mathrm{~nm}$ on Super-ACO for one mirror set) is limited to the mirror bandwith compared to the gain value. It will be widened on SRFELs with higher gain. The extention towards shorter wavelengths relies on the performances offered by the Ion Beam Sputtered mirrors in the UV.

The natural relative laser linewidth is of the order of $10^{-4}$. Linewidth narrowing down to $10^{-6}-10^{-7}$ was achieved on the VEPP3 FEL with an intra-cavity etalon [15]. It then improves the potentialities of SR FELs sources for spectroscopic applications. 
In addition, the first odd harmonics can produce a coherent emission, stimulated by the FEL, and could be extracted with a hole coupling in the front mirror of the optical cavity or a Brewster plate.

\subsubsection{Laser duration}

Operation at higher beam energies provides longer beam lifetimes and higher laser output powers (see Table I). The Super-ACO FEL at $800 \mathrm{MeV}$ leads to a laser duration of 10 hours, one order of magnitude greater than for the other existing FELs all operating below $500 \mathrm{MeV}$, and this forbids real use of these sources for experimental physics requiring a long functioning of the sources.

\subsubsection{Laser power}

The laser output power is proportional to the synchrotron radiation power, scaling linearly with the stored current and as the fourth power of the beam energy, and to the extraction efficiency (Brewster window, hole coupling or simply the mirror transmission as on the actual SR FEL (generally low). The Super-ACO FEL now provides at $800 \mathrm{MeV}$, $110 \mathrm{~mA}, 0.1 \mathrm{~W}$ of average power avaliable for the users, so more than two order of magnitude more than the other facilities and in its $1 \AA$ bandwidth, four orders of magnitude greater than the equivalent synchrotron radiation selected with a monochromator behind a $50 \mathrm{mrad}$ quartz window (in $\Delta \lambda=6 \mathrm{~nm}$ ).

\subsubsection{Polarization}

The FEL is polarized linearly in the horizontal plane, following the horizontal trajectory of the positrons in the vertical field undulator. Other polarizations may then be obtained with a Fresnel rhomb. The FEL could be circularly polarized with an helical undulator.

\subsubsection{Temporal structure}

The temporal structure of the FEL obviously mimics the one of the bunches stored in the ring (ns-ps pulses at a high repetition rate of the order of $10 \mathrm{MHz}$ ). The FEL micropulses are shorter than the synchrotron radiation pulses because the interaction occurs at the maximum of the electron density. The shortest FEL micropulses are obtained on UVSOR, with 15 ps FWHM, with short electron bunches due to a second harmonic $\mathrm{RF}$ cavity. The rather high repetition rate is not usual for conventional UV lasers.

In addition to its microtemporal structure, the FEL can also present a macrotemporal structure on the millisecond range depending on the detuning (longitudinal overlap between the electron bunches and the optical pulses). It is in fact the general situation observed for storage ring FELs, except on Super-ACO where it presents a stable regime for perfect synchronism. Great advantage can be taken from this so-called "continuous macrotemporal" structure, especially for the user experiments. It is unique in the Super-ACO FEL and results from the high stability of the storage ring, and probably from the use of positrons [16].

Q-switch operation can be performed, by an external gain modulation and it leads to a more stable pulse intensity and an increased peak power.

\subsubsection{Evolution versus detuning}

SR FELs operate over a given synchronization range, in which is observed different behaviours. On UVSOR, one distinguishes three zones : the central with a pulsed macrotemporal structure for maximum laser power and minimum laser duration. On Super-ACO, there are five area of operation, reported in table II. The perfect tuning (type 3) corresponds to the optimum laser parameters (shorter pulse duration and linewidth, higher power, CW operation) but presents some instability (laser micropulse jitter as great as $150 \mathrm{ps}$ and wavelength drift) [17], which are probably not the most desirable for the users. The type 1 and 5 correspond to a stabler laser (CW, less jitter and intensity fluctuations) but with a lower power and larger pulse duration and linewidth. On the contrary, a pulsed temporal structure at the ms range is observed in types 2 and 4), with consequently high intensity fluctuations, that really limits the FEL performances for users. 
Table II : stability of the laser parameters versus detuning

\begin{tabular}{|l|l|l|l|l|l|l|l|}
\hline type & $\begin{array}{l}\text { tuning } \\
\mid \Delta \mathrm{f}_{\mathrm{RF}}(\mathrm{Hz})\end{array}$ & $\begin{array}{l}\sigma \text { laser } \\
(\mathrm{FWHM})\end{array}$ & jitter & macro & power & $\Delta \lambda(\AA)$ & $\lambda$ drift \\
\hline $1-5$ & $>10$ & $70 \mathrm{ps}$ & small & $\mathrm{CW}$ & $<80 \%$ & $0.8-1.1$ & $<1 \AA$ \\
\hline $2-4$ & $5-10$ & $60-65 \mathrm{ps}$ & $<50 \mathrm{ps}$ & pulsed & $\approx 90 \%$ & $0.5-1.2$ & $2-3 \AA$ \\
\hline 3 & 0 & $60 \mathrm{ps}$ & $<150 \mathrm{ps}$ & $\mathrm{CW}$ & $\max$ & $0.4-1$ & $<1 \AA$ \\
\hline
\end{tabular}

\subsubsection{Stabilization of the FEL}

The perfect synchronisation between the optical pulses and the positrons must be adjusted to within $10^{-8}$. A longitunal feedback, detects at $300 \mathrm{~Hz}$ the longitudinal position of the laser micropulse compares it to that corresponding to perfect tuning, and readjusts the synchronization by a small change in the RF frequency. It allows to reduce the laser micropulse jitter, the intensity fluctuations down to $1 \%$ and the wavelength drift to less than $0.1 \AA$ [18].

\section{LINEAR ACCELERATOR FEL FOR THE UV-VUV RANGE}

\subsection{The UV Los Alamos FEL}

In 1993, the successful operation of the Los Alamos FEL in the UV answers to several technological challenges:

- photoinjector for the LINAC at $46 \mathrm{MeV}$, with a beam energy spread smaller than $1 \%$

- micro-undulator with a period of $1.4 \mathrm{~cm}$

- operation on the third harmonic with a gain of $12 \%$

The employed mirrors are multilayers of $\mathrm{HfO}_{2}$ and $\mathrm{SiO}_{2}$, with a transmission of $1 \%$. The laser operated between $369-380 \mathrm{~nm}$, in a relative bandwidth of $410^{-3}$, with a series of macropulses of $40 \mu$ s filled with $8-15$ ps micropulses, a laser risetime of $20 \mu$ s. The total average extracted power is of $0.7 \mathrm{~mW}$, with a transwerse laser mode waist of $0.12 \mathrm{~mm}$.

\subsection{Projects for the VUV range}

Two projects are under way in USA, one based on the harmonic generation from an external laser, and the second one in the optical cavity scheme.

In the DUV proposal at Brookhaven National Laboratory [20], a 3mJ/pulse Ti-Sa laser at $300 \mathrm{~nm}$ will produce coherent emission at $1000 \AA$ in an optical klystron where the period of the first (resp. second) undulator is adjusted to the input laser (resp. third harmonic), and this radiation will finally be amplified in a tapered undulator to provide 10 $\mathrm{mJ} /$ pulse. Chirping will allow very short pulses to be produced. The different elements of the project are in house.

On CEBAF is planned an $\mathrm{kW}$ UV FEL between $190-350 \mathrm{~nm}$ on a superconducting accelerator to perform industrial processing [21].

Of course, for these devices, the required good beam quality imposes the use of a photoinjector.

\section{PROSPECTS FOR X-RAY FELS}

In order to extend the FEL radiation towards the VUV and X-ray regions, high gain systems are required, especially considering the lack of very performant optics, eventhough the soft X-ray multilayers developed in astrophysics and synchrotron radiation community seem to be very promissing. Single pass devices are proposed, where the spontaneous emission occuring in the beginning of the undulator is sufficiently amplified along its travel inside the insertion device to lead to coherent radiation at the exit (Self Amplified Spontaneous Emission, or SASE). Theoretical calculations are carefully performed [22].

A more ambitious project proposes to use the SLAC linear accelerator for the production of SASE $1.4 \mathrm{GW}$ at $4.5 \AA$ and $40 \mathrm{GW}$ at $1.5 \AA$, with a very long undulator (up to $50 \mathrm{~m}$ ). The enhancement of the power, as compared to the present third generation 
synchrotron radiation will be of four order of magnitude. Further more, the use of a linear accelerateur allows to provide very short pulses, less than 1 ps. This source is the most ambitious one, but very promising with very short coherent X-ray pulses.

A second proposition on TRISTAN in Japan aims to instal a FEL around $30 \AA$ on a $180 \mathrm{~m}$ long by-pass at $4 \mathrm{GeV}$ with a coherence length of $2 \mu \mathrm{m}$ (compared to the $6 \mathrm{~mm}$ of the bunch). Harmonic generation is also considered [24].

\section{APPLICATIONS}

The first UV FEL has only being obtained in 1988 (compared to 1977 in the infrared), the first users experiments started later. They are now carried out on the Super-ACO FEL, because of its power, temporal structure, stability, polarization and duration for one beam injection. Several techniques can take advange of the FEL properties. This section reports only examples carried out on the Super-ACO FEL, the only source of this type presently used. Various different applications could also be developed.

\subsection{Time-resolved fluorescence experiments}

The feasibility of using the Super-ACO storage ring as a UV light source was demonstrated with the study of polarized fluorescence decays of the reduced nicotinamide adenine dinucleotide coenzyme (NADH) in aqueous solution, using the single photon counting (SPC) technique [5]. The FEL working point has been first optimized by monitoring the SPC apparatus function. A complete fluorescence experiment required about $30 \mathrm{~min}$ of data acquisition, during which the best integrated instrumental response had a FWHM of 110 ps. The measurements performed led to the unambiguous separation of two close lifetime components of $0.28 \mathrm{~ns}$ and $0.62 \mathrm{~ns}$ in the fluorescence decays of $\mathrm{NADH}$ at $20^{\circ} \mathrm{C}$, in good agreement with previous works. The thermodynamic parameters obtained from temperature studies show that the NADH fluorescence heterogeneity is consistent with the ground state folding equilibrium of the coenzyme, as characterized by many other spectroscopic techniques. From the fluorescence anisotropy decays, an apparent hydrodynamic radius of about $6 \AA$ is determined, while on the other hand, a large initial depolarization of the fluorescence indicates a fast independent motion of the nicotinamide ring. The quality of the collected data fully meets the requirements for the study of more complex systems such as fluorescent compounds bound to proteins or membranes. Further experiments on NADH at $280 \mathrm{~nm}$ and the study of the triptophane at $300 \mathrm{~nm}$ are planned. Time-resolved fluorescence techniques can also apply to solid state physics where higher intensities are required, on $\mathrm{CsPbCl3}$ crystals for example [25].

\subsection{Two-color experiments}

A storage ring FEL is really a unique tool for performing two-color experiments in all scientific domains for various phases of the matter (condensed, surface, gas), coupling both the laser source and synchrotron radiation (SR), taking advantage of the pulse to pulse natural synchronization, tunability of both FEL and SR (from the infra-red to the Xrays), their high repetition rate, and the FEL power . [26]. Because of the really wide range of SR and the improved compatibility of the Super-ACO FEL with the other users (see 2.1), one can now couple this FEL together with VUV or IR beamlines, and with various SR sources (bending magnets, undulators..) Varying the delay between the two sources is very promising. The FEL can be sent to the experimental site with an optical fiber or by a mirror transport line.

On a first application in surface physics [27], the surfave photovoltage (SPV) effect induced by the the Super-ACO FEL on a semi-conductor/metal (GaAs/Ag) interface has been probed by core level photoemission ( $3 \mathrm{~d}$ of $\mathrm{Ga}$ ) with synchrotron radiation from an undulator beamline. The SPV resulting from the creation of hole-electron pairs induced by the laser, modifies the electronic band structure at the interface, and the core level photoemission peaks are displaced. The studies will be completed with measurements versus the delay between the FEL and synchrotron radiation, the doping of GaAs and the temperature. 
A second application is planned in molecular physics [28] for the FEL-induced photoionisation of $\mathrm{He}^{*}$ : excited in the $2 \mathrm{p}$ level by SR. First tests were performed concerning the synchronization of the 2 pulses, and position of the beam in SA71. Advantage can be taken from the FEL tunability for the dtermination of the photionization cross-section. First results were obtained with a mode-locked synchronized Ar laser [29] for the photoionization of the $3 p$ level.

Other interesting experiments could involved the use of the FEL to induce photocarriers on HgCdTe materials, with an infrared detection with synchrotron radiation from the bending magnet A5 [30].

Finally, the applications of X-ray FEL sources has been considered during a workshop held at Stanford [31]. They cover the general use of X-ray synchrotron radiation, $\mathrm{X}$-ray lasers...

\section{CONCLUSION}

Free Electron Lasers appear to be very promising sources in the UV and VUV range, eventhough their development started later than the FELs in the infra-red, mainly because of the lower gain when going to shorter wavelength and less technological development for the optics. Nevertheless, the Super-ACO FEL demonstrated the quality of such sources for user, and the wide range of applications offered by two-color experiments coupling storage ring FEL and synchrotron radiation. The development of $\mathrm{X}$ ray $F E L$ is very challenging, both from the point of view of the technological requirements that are requested, but also by the new perspectives that can be offers with the enhanced brilliance and short pulses of these sources.

\section{REFERENCES}

[1] Deacon D.A.G., Elias L.R., Madey J.M.J., Ramian G.L., Schwettman H.Z. and Smith T.T. : Phys. Rev. Lett. Vol.38, pp 892-894, 1977

[2] W. Amersford Proceed. EPAC 94, London June 1994

[3] Billardon M., Elleaume P., Ortega J. M., Bazin C., Bergher M., Velghe M., Deacon D. A. G. and Petroff Y. Phys. Rev. Lett. 51, 1652 (1983)

[4] Kulipanov G.N., Pinaev I.V., Popik V.M., Skrinsky A.N., Sokolov A.S., Vinokurov N.A., Nucl. Instr. Meth., A296, 1-3 (1990)

[5] Couprie M. E., Tauc P., Merola F., Delboulbé A., Garzella D., Hara T., Billardon M. , Rev. of Scient. Instrum. 65 (5) 1485-1495 (1994)

[6] Couprie M. E., Garzella D., Billardon M. Europhysics Lett. 21 (9), 909-914 (1993)

[7] Yamada K., Yamazaki T., Sugiyama S., Tomimasu T., Ohgaki H., Noguchi T., Mikado T., Chiwaki M., Susuki R., Nucl. Inst. Meth. A318, 33 (1992)

[8] Yamazaki T., Yamada K., Sugiyama S., Ohgaki H., Sei N., Mikado T., Noguchi T., Chiwaki M., Suzuki R., Kawai M., Yokoyama M., Owaki K., Hamada S., Aizawa K., Oku Y., Iwata A., Yoshiwa M., Nucl. Instr. Meth., A331, 27 (1993)

[9] Takano S., Hama H., Isoyama G., UVSOR activity report 1992, p 4-7

Hama H., Yamazaki J. and Isoyama G. Nucl. Inst. Meth. A 341 (1994) 12-16

[10] "DELTA : a status report" DELTA group Univ. of Dortmund, Inst. of Physics 1990

[11] Litvinienko V., Madey J. M. J., Vinokurov N. Proceedings of the Particule Accelerator Conference Washington D. C. may 17-20 (1993)

[12] Couprie M.E., Ortéga J. M. "Lasers à Electrons Libres" Projet SOLEIL : Argumentation scientifique (Editions de Physique), Editeurs Chandesris D., Morin P. , Nenner I, , june 1993 p257-262

[13] Vinokurov N. A., Skrinsky A. M. Preprint INP77.59 Novosibirsk 1977

[14] Billardon M., Garzella D., Couprie M. E. Phys. Rev. Lett. 69, n $16,2368-2371$

[15] Couprie M. E., Gavrilov N.G., Kulipanov G.N. , Litvinienko V.N. , Pinaev I.V., Popik V. M.,Skrinsky A. N. , Vinokurov N. A. NIM A 304 (1991) 47-52

[16] Couprie M. E., Velghe M., Prazeres R., Jaroszynski D., Billardon M., Phys. Rev. A . 44, n², $130[-1315(1991)$

[17] Couprie M. E, V. Litvinenko, Garzella D., Delboulbé A., Velghe M., Billardon M. Nucl. Inst. Meth. A 331 (1993) 37-41

[18] "A longitudinal feedback for the Super-ACO Free Electron laser stability in the UV" M. E. Couprie, D. Garzella, T. Hara, J. H. Codarbox, M. Billardon Proceed. Of the 16 th Intern. FEL Conf. Stanford Univ. Aug. 1994 
[19] O'Shea P.G., Bender S.C., Byrd D.A., Early J.W., Feldman D.W., Fortgang C.M., Goldstein J. C., Newnam B. E., Sheffield R. L., Warren R.W., Zaugg T. J. Nucl. Inst. Meth. A 341 (1994)7-11

[20] "Milestone Experiments for single pass UV/Xray FELs" I. Ben-Zvi, Proceed. Of the 16th Intern. FEL Conf. Stanford Univ. Aug. 1994

[21] "Design of a high power UV FEL for Industrial processing" G. Neil Proceed. Of the 16th Intern. FEL Conf. Stanford Univ. Aug. 1994

[22] K. J. Kim Phys. Rev. Lett. 57 (1986) 1871

[23] "Performance Simulations and Parameter Optimization for High Gain Short Wavelength FEL amplifiers" G. Travish Proceed. Of the 16th Intern. FEL Conf. Stanford Univ. Aug. 1994; SLAC-PUB6312 LBL-33456 Aug 93

[24] "The TRISTAN Super Light Facility" Conceptual Design Report 1992 KEK Progress Report 921 A/M National Laboratory for High Energy Physics

[25] Beslky A.N., Vasil"ev, Mikhalin V. V., Gektin A.V., Shiran N. V., Rogalev A. L., Zinin E. I. Rev Scint. Instrum. 63 (1) Jan 1992 806-809

[26] Nenner I., Morin P., Meyer M., Lacoursière J. and Nahon L. Proceedings of the Maratea Conference, july 1992, to be published in NATO Asi Series; "Free Electron Laser Applications in the UV" 1988 Technical Digest Series, Vol. 4 (Optical Society of America, Washington DC, 1988)

[27] T. Hara , M. Marsi al "FEL induced surface photovoltage at Semi-conductor surfaces and interfaces on Super-ACO : a synchrotron radiation photemission study" presented at the first international FEL workshop Stanford 27-29 Aug 1994

[28] D. Garzella et al, "prospects for the first two-color experiments in atomic physics combining a UVstorage ring free electron laser with synchrotron radiation" presented at the first international FEL workshop Stanford 27-29 Aug 1994

[29] Lacoursière J., Meyer M., Nahon L., Morin P., Larzilliere M., "Time-resolved pump-probe photelectron spectroscopy of helium using a mode-locked laser synchronized with synchrotron radiation pulses" $^{\text {"1 }}$ to be published in NIM; Nahon L. et al., UVX'94 proceedings

[30] Carr G. L. et al Semicond. Sci. Technol. 8 (1993) 922-927

[31] "Workshop on Applications of Coherent X-rays" Stanford Feb. I2, 1994, SLAC-437, SLAC/SSRL0066 